\title{
AN INJECTOR AND LEBT FOR THE AAA ACCELERATOR*
}

\author{
Lash D. Hansborough, Joseph D. Sherman, and H. Vernon Smith, Jr. \\ Los Alamos National Laboratory, Los Alamos, New Mexico, 87545
}

\begin{abstract}
Design of an injector and LEBT for the AAA (Advanced Accelerator Applications) accelerator is based on the LEDA (Low-Energy Demonstration Accelerator) injector. This injector uses a microwave-driven generator to produce $75-\mathrm{keV}, 110-\mathrm{mA}$ dc and pulsed proton beams. The low-energy beam transport system (LEBT), comprised of two solenoid magnets and two steering magnets, delivers the beam to the RFQ match point. The LEDA injector has demonstrated $98 \%$ availability while operating at design parameters; however, it was not designed to minimize down time for maintenance, a critical consideration for AAA. Since the injector ion source and high-voltage column are the components most
\end{abstract}

control, and has provision for installation of a beam kicker. It is designed to be capable of replacement and return to full operation in less than four hours.

\section{INTRODUCTION}

A $\mathrm{cw}$ proton injector developed at LANL [1] is in routine use [2] for operation of the LEDA 6.7-MeV, 100$\mathrm{mA} \mathrm{cw}$ radio frequency quadrupole (RFQ) accelerator [3]. The LEDA injector uses a microwave-driven ion source [4] originated at Chalk River Laboratories. A 2.69-mlong low-energy beam transport (LEBT) delivers the required beam $(75-\mathrm{keV}, 110-\mathrm{mA} \mathrm{dc}, 0.20 \pi \mathrm{mm}$-mrad rms normalized) to the RFQ match point. An injector availability of $98 \%$ has been demonstrated at the design

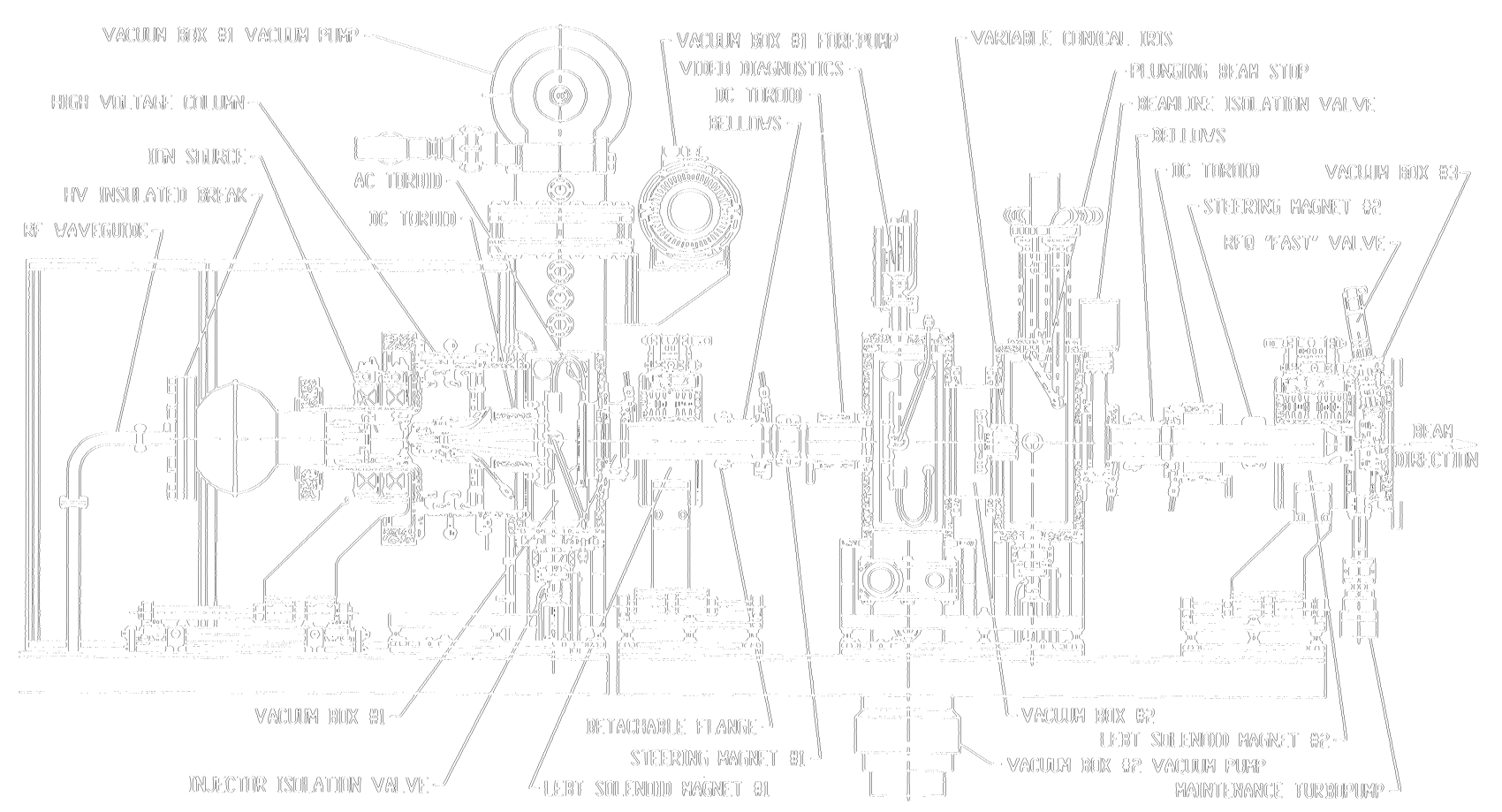

Figure 1. Line drawing of a 2.8-m-long AAA LEBT.

likely to degrade, the AAA injector ion source and high voltage column are designed to be removed and replaced as a unit without disturbing the LEBT components. The injector can be returned to full-current operation within 45 minutes. The AAA LEBT contains a plunging beam stop, a variable control iris for proportional beam current

- Work supported by the US DOE, NNSA and the Office of Nuclear Energy, Science, and Technology. parameters. The injector can also deliver a pulsed beam by pulsing the microwave power supply.

Minimal down time for maintenance is a critical consideration for AAA. An injector proposed for AAA [5] is shown in Figure 1: it is based on LANL experience with the LEDA injector and LEBT. The LEBT includes two solenoid magnets, three vacuum boxes, two steering magnets, and four beam pipes. Fabrication drawings of 
all components of the AAA injector and LEBT have been prepared at LANL.

The LEDA injector has demonstrated capability of quickly rolling on commercial hardware to the LEDA RFQ and reliably connecting within required alignment tolerances. The AAA injector is mounted on two tables using the same transportation system: one table is for components associated with beam production, and the other is for beam transport components.

Whereas the entire LEDA injector is located in the accelerator tunnel, all AAA injector power and water supplies are outside the tunnel, connected by quick disconnect devices to minimize the components in the accelerator tunnel which can degrade from radiation exposure or which require periodic maintenance. The only radiation at-risk components in the tunnel are video diagnostics.

\section{BEAM PRODUCTION}

AAA beam production begins in vacuum box \#1 (VB1) using components virtually identical to those in use on LEDA. Experience with LEDA shows the ion source and $\mathrm{HV}$ column are the components most likely to degrade and require periodic repair or replacement. After exposure to air, hours can be required to condition critical ion source and high voltage surfaces to return the injector to reliable operation. The AAA injector is designed to allow the replacement of vacuum box \#1 (VB1), containing a preconditioned ion source and high-voltage column assembly, as shown in Figure 2.

VB1 can be removed following closure of the vacuum box \#2 (VB2) beam line and vacuum pump isolation valves, and breaking vacuum on VB2. After removing a detachable beam pipe flange (in use on LEDA) and disconnecting the injector utilities, VB1 can be rolled back from the LEBT while maintaining vacuum in

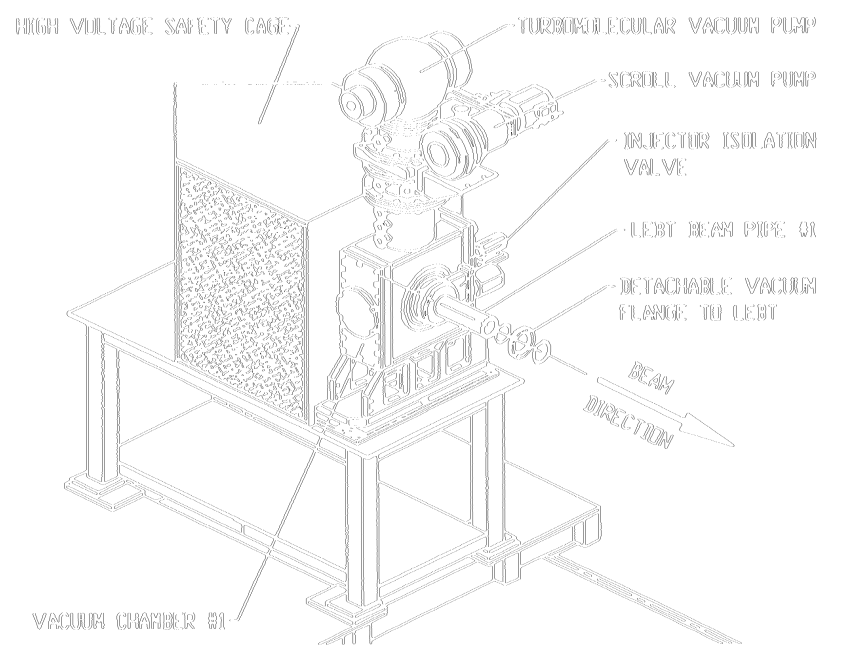

Figure 2. Vacuum Box \#1 disconnected from the LEBT. vacuum box \#3 (VB3) and without disturbing the alignment of any LEBT component. A replacement VB1 under vacuum is then rolled up to the LEBT with the injector isolation valve closed. Following installation of the beam pipe flange, VB2 is brought under vacuum. When the injector and beam line isolation valves are opened and injector utilities reconnected, the injector is ready for operation.

AAA injector replacement is scheduled to occur twice a year. It is anticipated that an efficient crew can return the injector to full current operation within 45 minutes, once access to accelerator tunnel is gained.

\section{LOW ENERGY BEAM TRANSPORT}

The AAA LEBT consists of components identical or similar to those used on LEDA. VB2 consists of two vacuum chambers connected by a spool. The upstream chamber is for diagnostics and vacuum pumping while the downstream chamber houses a plunging beam stop of the LEDA design. If the AAA beam is required to be turned off more quickly than can be accomplished by interrupting the microwave power supply to the ion source, a beam kicker may be required. A kicker magnet mounted in the upstream diagnostics box can deflect the beam onto the withdrawn plunging beam stop. The interbox spool can be easily lengthened to accommodate any kicked beam angle.

LEDA RFQ tests show minor but adjustable collimating of the beam is desirable to improve beam quality. During pulsed beam tests, a variable flat-plate iris was used on LEDA, but surfaces inclined to the beam are required to cope with $\mathrm{cw}$ beams. Figure 3 shows a simplified variable conical iris. The iris can fully close as eight cooled flaps fold inward and roll backward on ball

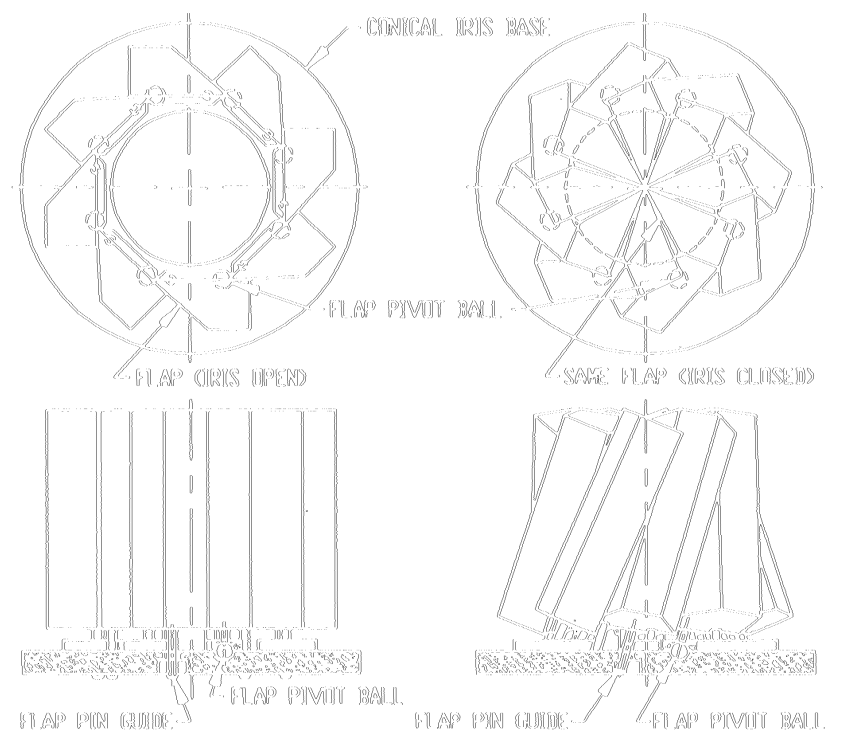

Figure 3. Variable conical beam iris. 


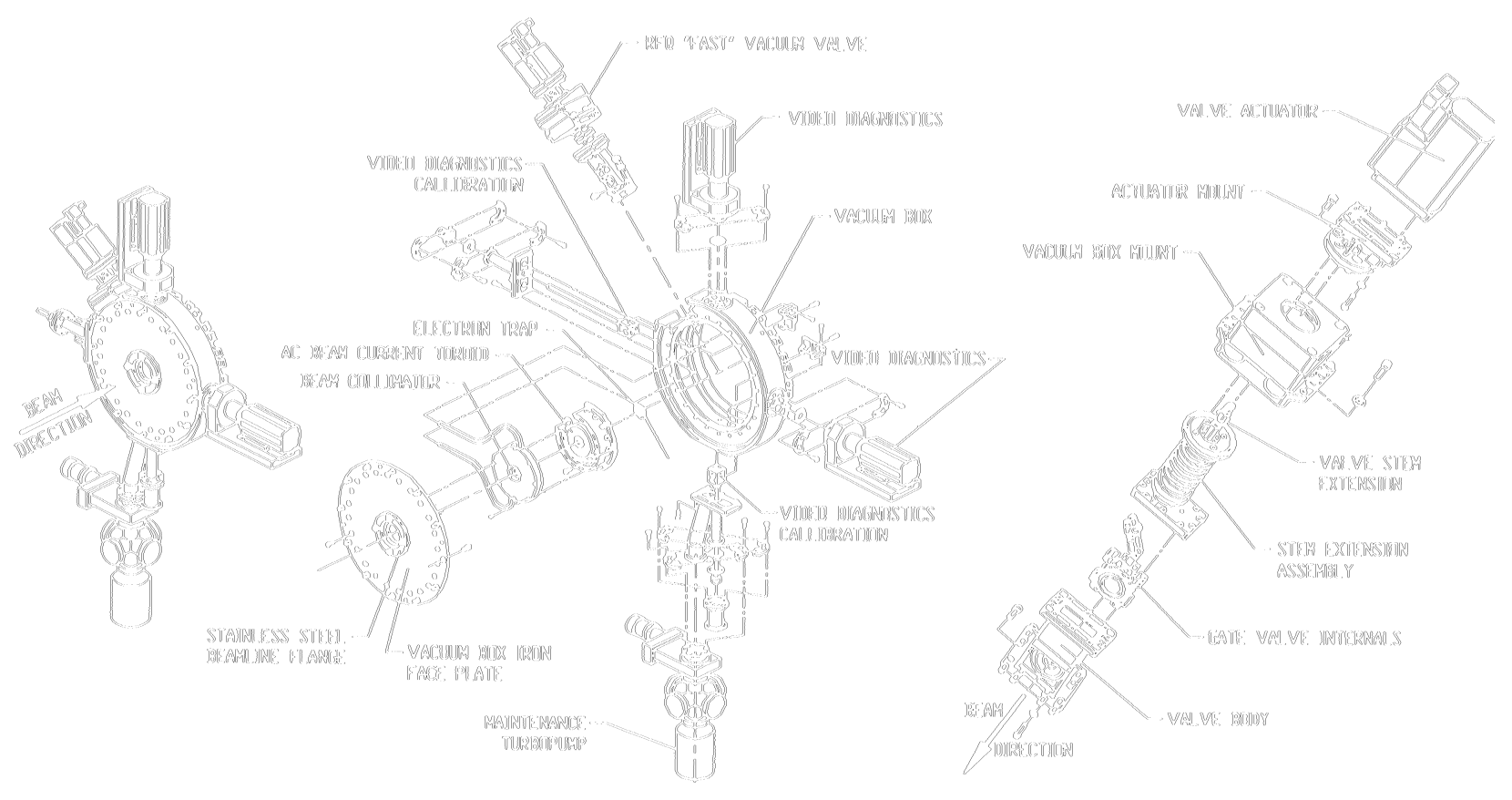

Figure 4. LEBT Vacuum Box \#3 (VB3).

Figure 5. RFQ "fast" valve.

pivots to form a collimator surface akin to the interior of a native-American teepee.

VB3 is the interface between the LEBT and the RFQ. The AAA VB3 (Figure 4) is similar to, but $1 / 3$ as long as the LEDA VB3 [6] which is no longer in use. The AAA VB3 incorporates an electron trap to improve beam transmission to the RFQ plus the same array of beam diagnostics as the LEDA VB3 except there is no DC beam current toroid. The AAA VB3 permits LEBT solenoid magnet $\# 2$ to be within $10.2 \mathrm{~cm}$ of the RFQ match point (vs. $15 \mathrm{~cm}$ in the present LEDA LEBT). The RFQ "fast" valve (Figure 5) is the same as one successfully used in the LEDA VB3: when shut, most VB3 components can be accessed while maintaining vacuum in the RFQ.

Experience with LEDA indicates little degradation of LEBT or VB3 components that might require periodic replacement; however, the AAA LEBT is designed for replacement without compromising the RFQ vacuum. After removal of VB1, the RFQ "fast" valve is closed and the beam line isolation valve opened to bring VB3 up to air. Pulling LEBT solenoid magnet \#2 back from VB3 exposes detachable beam pipe flange: this flange is removed and the LEBT rolled away, leaving VB3 attached to the RFQ. Reinstallation is done in reverse order. The AAA LEBT should be capable of replacement and return to full operation in less than four hours, an operation that may occur every two years.

\section{ACKNOWLEDGMENTS}

The authors wish to acknowledge Luis Ramirez whose graphical artistry aided this work.

\section{REFERENCES}

[1] J.D. Sherman, et al., "A DC Proton Injector for Use in High-Current CW Linacs," Proc. EPAC98 (Stockholm, 22-26 June 1998) pp. 1424-1426.

[2] J.D. Sherman, et al., "A 75-keV, $145 \mathrm{~mA}$ Proton Injector," Proc. ICIS2001, (Oakland, 3-7 September 2001) to be published.

[3] L.M. Young, et al., "High-Power Operations of LEDA," Proc. LINAC2000 (Monterey, 21-25 August 2000) pp. 336-340.

[4] T. Taylor and J.F. Mouris, Nuclear Instrum. and Methods in Phys. Research A336, 1 (1993).

[5] D.W. Christiansen, et al., "Injector System Design Description," PPO-A11-G-X-00001, Rev. B, September 2000, in "Accelerator Production of Tritium Facility Design Description," PPO-POOFDD-X-00001.

[6] L.D. Hansborough, et al., "Mechanical Engineering of a 75-keV Proton Injector for the Low-Energy Demonstration Accelerator," Proc. PAC97 (Vancouver, 12-16 May 1997) pp. 2740-2742. 